\title{
Improved particle position accuracy from off-axis holograms using a Chebyshev model
}

\author{
JoHAN ÖHMAN* AND MIKAEL SJÖdAHL \\ Experimental Mechanics, Luleå University of Technology, SE-971 87 Luleå, Sweden \\ *Corresponding author: johan.ohman@ltu.se
}

Compiled November 9, 2017

\begin{abstract}
Side scattered light from micrometer sized particles is recorded using an off-axis digital holographic setup. From holograms a volume is reconstructed with information about both intensity and phase. Finding particle positions are non-trivial since poor axial resolution elongates particles in the reconstruction. To overcome this problem the reconstructed wavefront around a particle is used to find the axial position. The method is based on the change in sign of the curvature around the true particle position plane. The wavefront curvature is directly linked to the phase response in the reconstruction. In this paper we propose a new method of estimating the curvature based on a parametric model. The model is based on Chebyshev polynomials and is fitted to the phase anomaly, compared to a plane wave, in the reconstructed volume. From the model coefficients, it is possible to find particle locations. Simulated results shows increased performance in the presence of noise compared to the use of finite difference methods. The standard deviation is decreased from $3-39 \mu \mathrm{m}$ to $6-10 \mu \mathrm{m}$ for varying noise levels. Experimental results shows a corresponding improvement where the standard deviation is decreased from $18 \mu \mathrm{m}$ to $13 \mu \mathrm{m}$. ๑ 2017 Optical Society of America
\end{abstract}

OCIS codes: (090.1995) Digital holography; (290.5850) Scattering, particles; (290.4020) Mie theory; (100.0100) Image processing

http://dx.doi.org/10.1364/ao.XX.XXXXXX

\section{INTRODUCTION}

Digital holography is a suitable technique for tracking microparticles since it records 3D information. Holography was originally proposed by Gabor in 1948 [1] and its digital version is usually attributed to Goodman [2]. Extracting information about particle fields is a natural application of digital holography as 3D information about position, size and morphology are recorded in the hologram. Applications include studies of; flow phenomena using holographic particle velocimetry (HPIV) [3], tracking microorganisms [4], characterizing aerosols [5-7], dynamic behavior of combustion fuel particles [8,9] and air bubbles [10], to mention a few. There are two main configurations of holographic setups used for particle analysis. In in-line holography the illumination wave is also used as reference wave. Here the scattered light from particles interferes with the un-scattered light in the forward direction. This is a very simple and compact setup, but it has disadvantages in that only the forward direction can be used and that a twin image and zero-order terms disturb the reconstruction. The other main configuration is off-axis holography that uses a separate reference wave with an off-axis angle. This setup is more complex but with it, it is possible to remove the twin image and zero-order content from the image of interest. Any imaging direction can be used with this setup, not only the forward direction. In this investigation the off-axis configuration is utilized as it allows for a general registration direction. In digital holography the hologram is recorded on a CCD or CMOS detector and is numerically reconstructed to obtain 3D information [11, 12]. For low numerical apertures the resolution in the reconstruction along the optical axis is poor compared to the lateral one [13], therefore particles appear elongated. To find the correct axial particle position a metric of some kind is needed. Previously reported metrics are based on intensity [14-16], which are most common, or are based on the whole complex field $[17,18]$. It is also possible to solve the inverse problem directly from the recorded hologram $[19,20]$. This is however more time consuming and is therefore unsuitable for large particle sets.

We have in a previous paper presented a metric that is based on the wavefront curvature of the scattered light [21]. This metric utilizes the fact that scattered light from a particle converges towards its true position and thereafter diverges. The change from a converging to a diverging wave is found by tracking the wavefront curvature. One significant advantage with a phase based metric is that it does not have an offset 
that is present in intensity based metrics [22]. The wavefront curvature was in our previous paper estimated from phase gradients that were computed using finite differences. This was a simple way to estimate the curvature; unfortunately in the presence of noise the precision decreases rapidly due to the noise enhancement. In this paper we propose a method that parameterizes the phase anomaly compared to a plane wave, without using finite differences. The method consists of fitting the phase anomaly to a three-dimensional polynomial model, and from the polynomial coefficients determine the particle position. By using a model we reduce the effect of noise. The rest of the paper is structured in the following way: In section 2, theory regarding particle scattering, digital hologram formation and reconstruction are presented. The proposed polynomial model is also outlined. In section 3 the setup is described along with the simulations and measurements performed. In section 4 the results from both simulations and measurements are presented and discussed. Finally, the paper is summed up in section 5 and conclusions are presented.

Portions of this work were presented at the Digital Holography \& 3-D Imaging conference in 2017, Paper M4A.3.

\section{THEORY}

\section{A. Particle Holograms}

Light incident on a particle generates an angular dependent outwards propagating scattered field. Consider the geometry sketched in Fig. 1. The particle is located at the origin of a right handed Cartesian coordinate system and is illuminated by a plane wave propagating along the $y$-axis. Detection is done along the vicinity of the $z$-axis that hence is denoted as the optical axis. We denote the Jones vectors of the incident light $\bar{E}_{i}$ and the scattered light $\bar{E}_{s}$, respectively. For spherical micrometer sized particles, the Lorenz-Mie theory is a suitable scattering model [23]. The theory is most commonly expressed with respect to the scattering plane, this is the plane spanned by the illumination and observation directions, denoted $\bar{s}_{i}$ and $\bar{s}_{S}$ respectively, see Fig.1. The incident and scattered fields are then expressed in polarization components parallel and perpendicular to the scattering plane. These components are denoted $E_{i \perp}, E_{i \|}$ for the incident light, and $E_{S \perp}, E_{S \|}$ for the scattered light, respectively. The relation between these components is as follows:

$$
\bar{E}_{S}=\left(\begin{array}{c}
E_{S \perp}(\theta) \\
E_{S \|}(\theta)
\end{array}\right)=\frac{e^{i k(r-z)}}{-i k r}\left(\begin{array}{cc}
S_{1}(\theta) & 0 \\
0 & S_{2}(\theta)
\end{array}\right)\left(\begin{array}{c}
E_{i \perp} \\
E_{i \|}
\end{array}\right),
$$

where $S_{1}(\theta)$ and $S_{2}(\theta)$ are the angular dependent scattering coefficients, $\theta$ is the in-plane angle, $\mathrm{z}$ is the position along the optical axis and $r=\sqrt{x^{2}+y^{2}+z^{2}}$ is the distance from the particle. For the derivation of equation (1) and the full expression for the scattering coefficients we refer the reader to [23].

In digital particle holography the object light $\bar{E}$, given by equation (1) and a reference wave given by

$$
\bar{R}=\left(\begin{array}{c}
R_{\|} \\
R_{\perp}
\end{array}\right)
$$

interfere on the detector to form a digital hologram. Only components with mutual polarization interfere. In an off-axis holographic setup the object light, in this case the light scattered from particles, and the reference light are assumed to be separated in

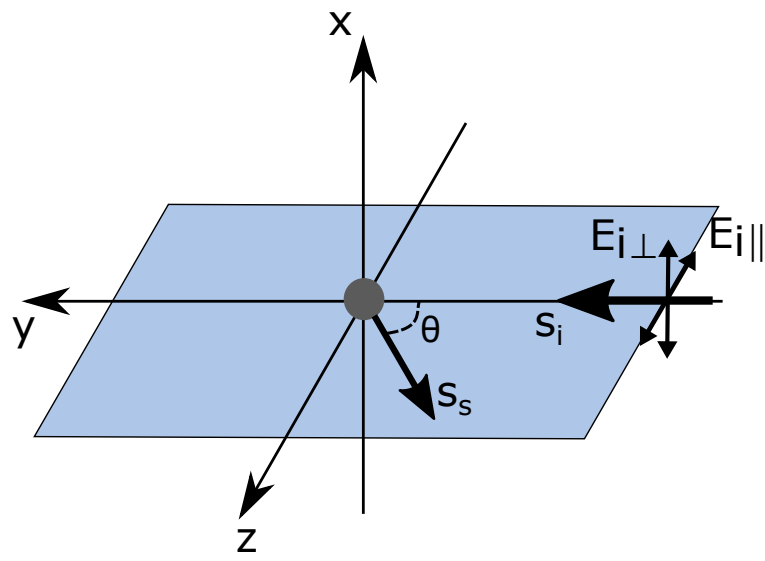

Fig. 1. Scattering geometry with the defined scattering plane, spanned by vectors $s_{i}$ and $s_{s}$. The polarization is expressed on component form parallel and orthogonal to this plane.

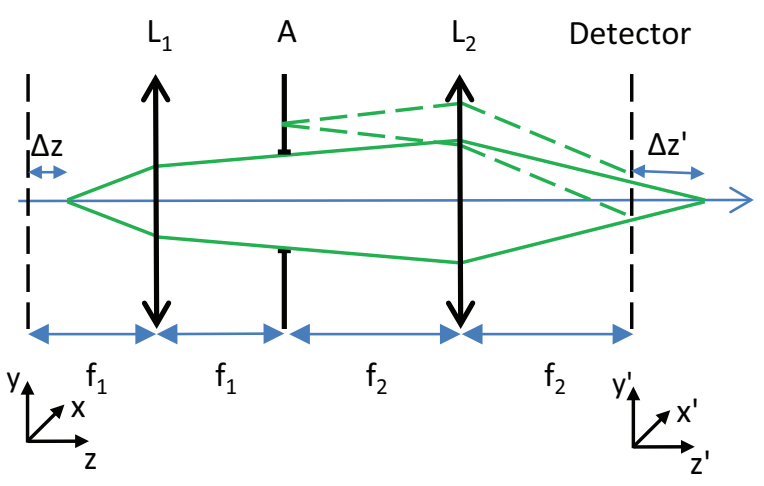

Fig. 2. Telecentric imaging system consisting of lenses $L_{1}$ and $L_{2}$ and aperture $A$. The solid rays is the object light and the dashed rays are the reference light. The dashed black lines indicates the detector in image space and its conjugate plane in object space.

the angular spectrum [11]. In our setup the light is recorded by the two-lens telecentric imaging system, depicted in Fig. 2. The magnification $M$ between object and image space is then given by $M=f_{2} / f_{1}$, where $f_{1}$ and $f_{2}$ are the focal lengths of lens $L_{1}$ and lens $L_{2}$, respectively. We choose to denote the coordinate system in the object space by $(x, y, z)$ and the coordinates in the image plane on the detector by $\left(x^{\prime}, y^{\prime}, z^{\prime}\right)$, respectively. The imaging system then maps from the object plane to the image plane. The coordinates are related by the magnification $M$ of the system as $x^{\prime}=-M x, y^{\prime}=-M y$ and $\Delta z^{\prime}=M^{2} \Delta z$, where $\Delta z$ and $\Delta z^{\prime}$ are the defocus distances in object and image space, respectively. The reference wave is an off-axis point source in the aperture plane that is reshaped by the lens $L_{2}$ to become a plane wave. In these notations the intensity on the detector for an arbitrary polarization becomes: 


$$
\begin{aligned}
I\left(x^{\prime}, y^{\prime}\right) & =\left|\bar{E}\left(x^{\prime}, y^{\prime}\right) \bar{R}\left(x^{\prime}, y^{\prime}\right)\right|^{2} \\
& =\left|E_{\|}\left(x^{\prime}, y^{\prime}\right)\right|^{2}+\left|E_{\perp}\left(x^{\prime}, y^{\prime}\right)\right|^{2}+\left|R_{\|}\left(x^{\prime}, y^{\prime}\right)\right|^{2} \\
& +\left|R_{\perp}\left(x^{\prime}, y^{\prime}\right)\right|^{2}+E_{\|}\left(x^{\prime}, y^{\prime}\right)^{*} R_{\|}\left(x^{\prime}, y^{\prime}\right) \\
& +E_{\perp}\left(x^{\prime}, y^{\prime}\right) R_{\perp}\left(x^{\prime}, y^{\prime}\right)+E_{\|}\left(x^{\prime}, y^{\prime}\right) R_{\|}^{*}\left(x^{\prime}, y^{\prime}\right) \\
& +E_{\perp}\left(x^{\prime}, y^{\prime}\right) R_{\perp}^{*}\left(x^{\prime}, y^{\prime}\right),
\end{aligned}
$$

where the first four terms are the intensity of each polarization component of the object wave and reference wave, respectively. The fifth and sixth terms are interference between the conjugate object wave and reference wave, for each polarization respectively. Likewise, the seventh and eighth terms are the interference terms between the object wave and conjugate reference wave, for each polarization respectively. By controlling the offaxis angle of the reference wave and the aperture size in the system one can ensure no overlap between interference terms in the Fourier domain. Fig. 3a shows the recorded intensity on the detector for a simulated hologram. The particle is defocused a distance $\Delta z=2000 \mu \mathrm{m}$.

\section{B. Reconstruction}

The hologram captured by the detector has to be reconstructed before further analysis is done. It is possible to extract individual interference terms when using off-axis holography since they are separated into different lobes in the Fourier domain. The four first terms in equation (3), i.a the intensity components, are located in a central lobe. The four interference terms have each a separate side lobe in a well configured system. The interference term of interest is extracted by applying a rectangular mask around the corresponding lobe, removing all content outside it. If the reference wave $\bar{R}$ is a tilted plane wave it will scale the object wave and add a phase gradient proportional to the off-axis angle. It is possible to remove the phase gradient by re-centering the lobe after the filtering process. By doing so $R$ reduces to a scalar depending on the reference wave intensity. Because of this we drop the $R$ from now on, hence the complex amplitude $\tilde{E}\left(f_{x}^{\prime}, f_{y}^{\prime} ; z_{D}^{\prime}\right)$ is known in Fourier space for the component of interest. Where $\Delta z_{D}^{\prime}$ is the axial position of the detector in image space. It is now possible to compute the field at an axial position $z^{\prime}=z_{D}^{\prime}+\Delta z^{\prime}$ from the detector with the use of the angular spectrum method [11], the field becomes:

$$
E\left(x^{\prime}, y^{\prime}, z^{\prime}\right)=\mathscr{F}^{-1}\left[\tilde{E}\left(f_{x}^{\prime}, f_{y}^{\prime} ; z_{D}^{\prime}\right) \exp \left(-j k s_{z}^{\prime} \Delta z^{\prime}\right)\right]
$$

where $\mathscr{F}^{-1}$ is the $2 \mathrm{D}$ inverse Fourier transform, $k=\frac{2 \pi}{\lambda}$ is the wave number, and $s_{z}^{\prime}=\sqrt{1-\left(\lambda f_{x}^{\prime}\right)^{2}-\left(\lambda f_{y}^{\prime}\right)^{2}}$ is the directional cosine component in the z-direction. By reconstructing a stack of images separated a distance $\Delta z^{\prime}$ a discrete volume is produced. This reconstruction process creates a $3 \mathrm{D}$ volume from a $2 \mathrm{D}$ hologram. The volume contains information about both the amplitude and phase from which 3D particle positions can be found using a suitable metric.

The basic principle of our metric is that the reconstructed wave from a particle converges towards its position and thereafter diverges. A converging and a diverging wave have different sign of curvature, so the plane where the curvature changes sign is then the plane where the particle is located. The wavefront curvature can be obtained from the phase in the reconstructed volume. The phase in the volume oscillates quickly
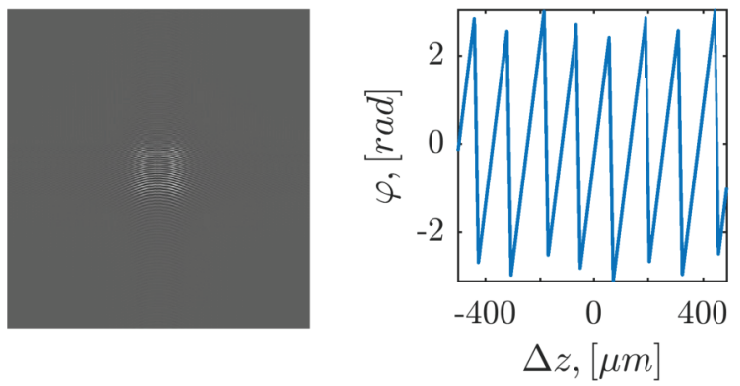

(a)

(b)

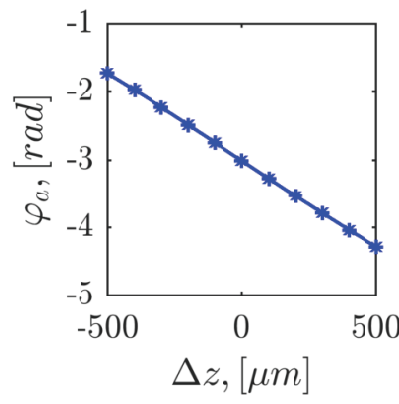

(c)

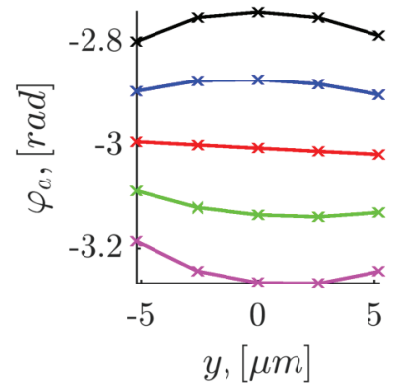

(d)
Fig. 3. Different properties in the reconstruction. (a) is the intensity on the detector for a simulated hologram, (b) is the reconstructed phase along the center of the particle, (c) is the corresponding phase anomaly to (b). (d) shows the wavefront curvature at $\Delta z=-100 \mu m$ (black), $-50 \mu m$ (blue), $0 \mu m$ (red), $50 \mu \mathrm{m}$ (green), $100 \mu \mathrm{m}$ (magenta). In both figures (c) and (d) the solid lines are the polynomial fit and the markers the simulated data.

along the optical axis, much faster than the step length $\Delta z^{\prime}$ in the reconstruction. This is problematic since it then contains discontinues in the z-direction making it unsuitable to fit it to a polynomial model. Fig. $3 b$ shows the reconstructed phase along the $\mathrm{z}$-direction along the center of the particle. Aliasing effects are present causing the oscillations to appear slower than they really are. By instead comparing the phase to a plane wave situated in the same plane it becomes possible to compare the data between two planes. This phase anomaly is calculated as follows:

$$
\varphi_{a}\left(x^{\prime}, y^{\prime}, z^{\prime}\right)=\angle\left[E\left(x^{\prime}, y^{\prime}, z^{\prime}\right)\right]-k z^{\prime},
$$

where $k$ is the wave number. We will from now on use the phase anomaly $\varphi_{a}$ instead of the pure phase since it is a smoother function more suitable for fitting. We now have two sets of information, the 3D intensity distribution $I\left(x^{\prime}, y^{\prime}, z^{\prime}\right)=\left|E\left(x^{\prime}, y^{\prime}, z^{\prime}\right)\right|^{2}$, and the 3D phase anomaly $\varphi_{a}\left(x^{\prime}, y^{\prime}, z^{\prime}\right)$, from which the particle position can be estimated.

\section{Chebyshev Polynomial Model}

It is assumed that the phase anomaly around a single particle only depends on the response from that particle. Only a small volume around a potential particle is therefore used in the parameterization. This assumption holds in general if the particle distribution is sparse. Potential particles are found from a course intensity based search where voxels with intensity over a defined threshold is chosen. 
For a single particle the phase anomaly, $\varphi_{a}$ is fitted to a model based on Chebyshev polynomials of the first kind [24]. Chebyshev polynomials are chosen as base functions for the parameterization for two reasons. The first is that they are orthonormal in a Cartesian coordinate system and therefore suitable for computations in a rectangular volume. Secondly, terms of different orders are associated with properties in the imaging process when using small to moderate numerical apertures. For instance, first-order terms are related to a spatial shift in direction and second-order terms are related to defocus. The modeled phase anomaly $\hat{\varphi}$ in three dimensions is defined from one dimensional Chebyshev polynomials. The polynomials are defined in the interval $[-1,1]$ and the coordinates $\left[x^{\prime}, y^{\prime}, z^{\prime}\right]$ are rescaled to normalized coordinates $[\epsilon, \eta, \zeta]$ in this domain. The $3 \mathrm{D}$ polynomial is then as follows:

$$
\hat{\varphi}_{a}(\epsilon, \eta, \zeta)=\sum_{i=0}^{n} \sum_{j=0}^{n} \sum_{k=0}^{n} a_{i j k} T_{i}(\epsilon) T_{j}(\eta) T_{k}(\zeta),
$$

where $T_{i}, T_{j}$ and $T_{k}$ are Chebyshev polynomials of order $i, j, k$, respectively, and $a_{i j k}$ are the associated coefficients for each base function triplet. Equation (6) can be rewritten on matrix form as:

$$
\hat{\varphi}_{a}(\epsilon, \eta, \zeta)=\mathbf{T} a .
$$

The objective is then to find the set of estimated coefficients $\hat{a}_{i j k}$ that best represents the measured phase anomaly $\varphi_{a}$. We minimize the squared error between the measured data, $\varphi_{a}$ and the estimated data, $\hat{\varphi}_{a}$, from the polynomial model. The coefficients are then the solution to the following minimization problem:

$$
\hat{a}_{i j k}=\underset{a}{\operatorname{argmin}} \sum W\left(\varphi_{a}-\hat{\varphi}_{a}\right)^{2}
$$

where $\mathrm{W}$ are weights that influence how much each data point contribute to the estimation. We chose the intensity of the scattered light as these weights. This ensures that only data points where we have light influence the estimation. The solution to this minimization problem is:

$$
\hat{a}=\left(\mathbf{T}^{T} W \mathbf{T}\right)^{-1} \mathbf{T}^{T} W \varphi_{a},
$$

which is most efficiently calculated using Gauss elimination. To ensure that the estimated coefficients, $\hat{a}_{i j k}$ in equation (6), represent the phase anomaly correctly a sufficiently high polynomial degree $n$ is needed. To find this value scattered light from a single particle is simulated using equations (1) and (3), respectively. For the full description of the simulation implementation we refer the reader to [21]. The simulated hologram is reconstructed according to section 3 . In the reconstructed volume the parameterization is performed and the mean squared error (MSE) per voxel is evaluated for different values of $n$. The result is shown in Fig. 4. A good fit is obtained for $n=4$ where the mean error is approximately $10^{-3} \mathrm{rad} /$ voxel. Using higher orders does not significantly improve the fit. Therefore a fit with $n=4$ is assumed sufficient and will be used further on in this paper. The phase anomaly corresponding to Fig. $3 \mathrm{~b}$ is shown in Fig. 3c. The markers are the simulated data and the solid line is the Chebyshev polynomial fit. Fig. 3d shows phase anomaly cross-sections in the $y$-direction for different z-positions. Here the change in wavefront curvature becomes apparent.

The three dimensional data is now represented by the coefficient array $a_{i j k}$. The relationship between the elements in the array and information about the particle is now investigated.

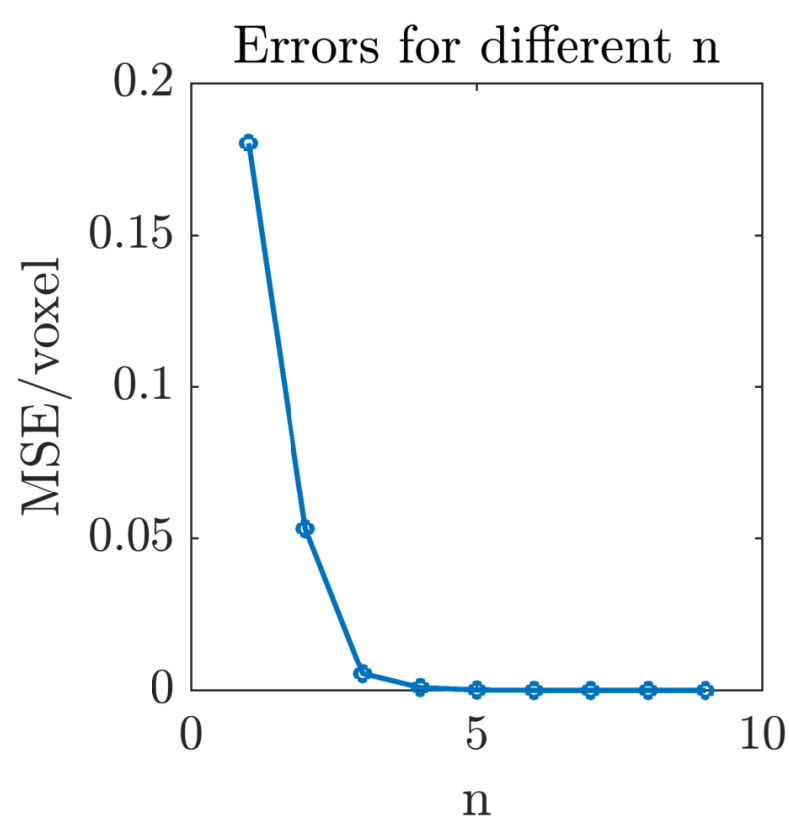

Fig. 4. Mean square error (MSE) per voxel for different orders of $n$.

A single particle is simulated and coefficients $\hat{a}$ are estimated for different axial positions by translating the computational box along the optical axis. The dimension of the box used is $5 \times 5 \times 21$ voxels. All coefficients are investigated and as an example the results for the coefficients $a_{200}$ and $a_{020}$ are shown in Fig.5. The coefficient $a_{200}$ has a linear behavior around the particle and becomes zero at the particle position. $a_{200}$ is therefore used as a metric to find the axial position of the particle. This coefficient corresponds to the quadratic term in the direction perpendicular to the scattering plane, so it describes the curvature of the wavefront in that direction. When $a_{200}$ becomes zero, the wavefront changes curvature. This behavior is consistent with the findings in [21]. The corresponding coefficient $a_{020}$, that represents the quadratic term in the direction parallel to the scattering plane, is also shown in Fig. 5. It shows a similar behavior but is slightly shifted compared to $a_{200}$. Therefore only $a_{200}$ will be used as a metric.

To use the metric on a set of particles recorded in a single hologram some practical considerations has to be made. First we need to segment particles from each other, this is done by applying a threshold on the intensity in the volume. From the binary volume connected voxels are clustered and labeled. The point with maximum intensity has a slight off-set from the particle position, but is still in the vicinity [22]. This point is therefore a good starting point when searching for the plane where $a_{200}$ becomes zero. A small 3D domain is formed around the maximum intensity point and the Chebyshev model is evaluated in this domain. The position where $a_{200}$ becomes zero is then the position of the particle. This position is found by computing the zero-crossing using linear interpolation. It is important that the particles are sparse so that only scattered light from an individual particle appear in the 3D domain. The whole procedure for obtaining the axial position of particles from a hologram can therefore be summarized in the following steps:

1. Reconstruct a three dimensional volume using the angular 


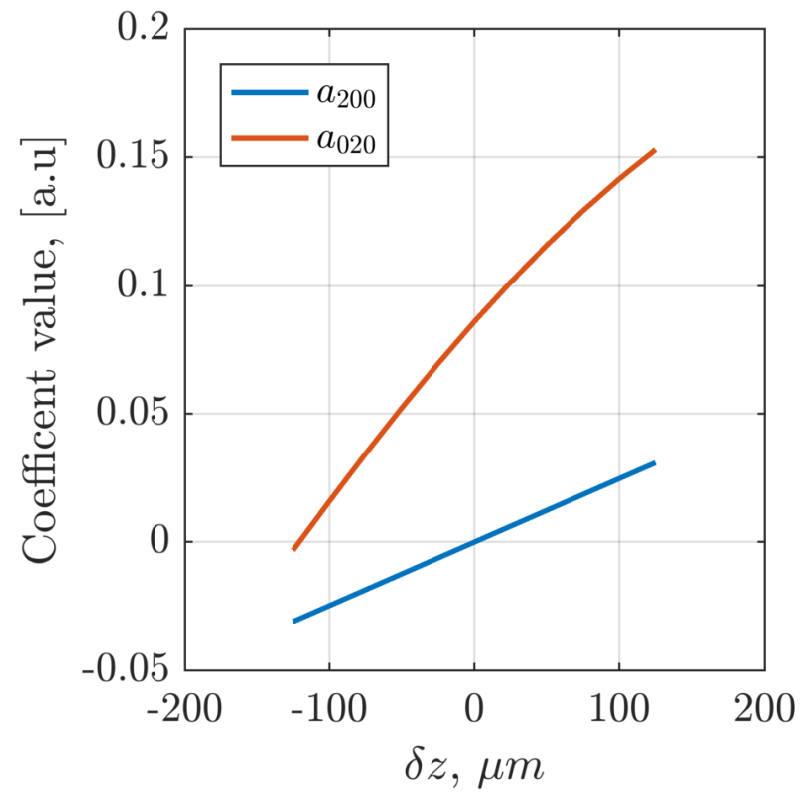

Fig. 5. Evaluation of the square terms $a_{200}$ and $a_{020}$ around the particle location.

spectrum method.

2. Apply a threshold on the intensity in the volume to obtain a binary volume.

3. Automatic segmentation of each particle from the binary volume.

4. Find the point of maximum intensity for each particle.

5. Evaluate the coefficient $a_{200}$ in a small domain around the point of maximum intensity for each particle.

6. The axial location where the coefficient $a_{200}$ becomes zero is stored as the position of each particle.

\section{EXPERIMENTS}

\section{A. Setup}

Consider the setup shown in Fig.6. A 532 nm Nd:YAG continuous laser with a power of $85 \mathrm{~mW}$ is used for illumination. The light is polarized parallel to the scattering plane. This polarization is chosen to reduce Rayleigh scattering from inhomogeneous parts in the sample. The light is split into two parts using a 50/50 beam splitter (BS). The first part of the light is expanded by the lenses L1 and L2 with focal lengths $f=150 \mathrm{~mm}$ and $f=-20 \mathrm{~mm}$, respectively. The expanded light illuminate the sample that consists of a $23 \times 23 \times 23 \mathrm{~mm}^{3}$ silicon cube with spherical particles molded into it. The particles have an average diameter of $10 \mu \mathrm{m}$ and the refractive indices of the silicon and particles are 1.4 and 1.5, respectively. The concentration of particles is low so that the imaged scene is sparse, only a few particles are present. The cube is placed so that only scattered light from the particles are imaged. There could be internal variations of the refractive index in the silicone cube that affects the experimental results compared to if the particles were imaged in air. These will then show up as an increase in random

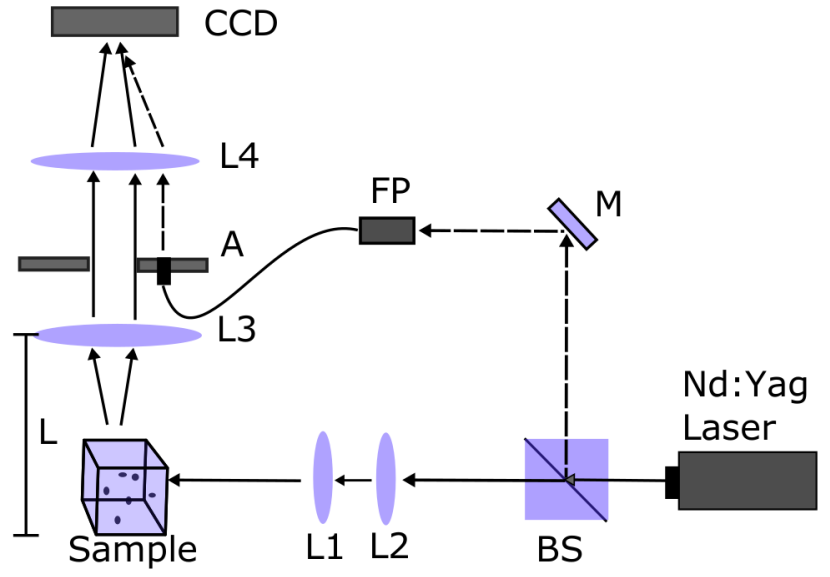

Fig. 6. Experimental setup used in the recordings. BS is a beam splitter, L1 is a $f=150 \mathrm{~mm}$ lens, L2 is a $f=-20 \mathrm{~mm}$ lens, L3 is a $f=60 \mathrm{~mm}$ lens, L4 is a $f=80 \mathrm{~mm}$ lens, $\mathrm{A}$ is the aperture, $\mathrm{M}$ is a mirror and $\mathrm{FP}$ is a fiber port.

error. Scattered light from the particles is recorded using a telecentric imaging system, formed by lenses L3 and L4 at a $90^{\circ}$ angle. Lenses L3 and L4 have focal lengths $f=60 \mathrm{~mm}$ and $f=80 \mathrm{~mm}$, respectively. This corresponds to a system magnification of $M=1.33$. A $3 \times 3 \mathrm{~mm}^{2}$ aperture is used to band limit the image. It is placed in the joint focal plane between lenses L3 and L4 making the system telecentric. The other light part from the beam splitter (BS) is used as the reference wave. It is focused into a polarization maintaining fiber using a mirror (M) and a fiber port (FP). The fiber end is placed in the aperture plane. By doing so we ensure that the reference light becomes a point source in the Fourier domain. In the back focal plane of lens L4 a detector (Sony XCL 5005) is placed to record the hologram. It has $2448 \times 2048$ pixels and a pixel pitch of $3.45 \mu \mathrm{m}$ giving the detector a total size of $8.48 \times 7.1 \mathrm{~mm}$. The images are stored in 8 bit format. The fiber has a numerical aperture of 0.12 which is sufficient to cover the whole detector. The numerical aperture of this system is $N A_{0}=0.025$ in the object plane and $N A_{1}=0.0187$ in the image plane, respectively. Since the sample is a silicon cube the effective numerical aperture is even smaller since the silicon-air boundary will refract the scattered light. The effective numerical becomes

$$
N A_{0}^{\prime}=\frac{N A_{0}}{n_{\text {silicon }}} \approx 0.0179
$$

\section{B. Simulations and measurements}

To compare how the parametric Chebyshev method compares to the previously reported finite difference method a large set of simulations are performed. Once again we use the simulation routine described in [21], that is an implementation of equations (1) and (3) respectively. Simulation properties are set as described in section 3.A. Some assumptions are made; like that the illumination is a perfect plane wave and that particles are identical perfect spheres with a diameter of $10 \mu \mathrm{m}$. 10 holograms each containing 20 particles located at random lateral and axial positions are simulated. Since the axial positions are known it is 
easy to compute the error for each particle. The standard deviation, false positive ratio and false negative ratio are computed. This is repeated for different noise levels ranging from 0 to 4 percent of the maximum intensity.

For experimental measurements the backside of the silicon cube is placed at approximately $L=60 \mathrm{~mm}$ in-front of the imaging system so that the focus plane is inside the sample cube. Unlike in simulations, the true particle position is not known. Therefore are several holograms of stationary particles recorded in series. Particle positions are evaluated with the method outlined in section 4 from the holograms. A set of positions for five different particles are then obtained. For each particle the axial mean position is estimated, by subtracting this value from the recorded positions the associated error is computed. From this data the standard deviation is calculated.

\section{RESULTS AND DISCUSSION}

The resulting standard deviations from simulations are shown in Fig.7 as a function of noise ratio. Data for both the Chebyshev model and the finite difference method is presented. It is clear that by using the Chebyshev model the standard deviation drastically decreases for larger noise ratios. At the highest simulated noise ratio of $4 \%$ the standard deviation is reduced from $39 \mu \mathrm{m}$ to $10 \mu \mathrm{m}$, an improvement of about 4 times. This can be explained by the fact that the finite difference is influenced by the noise in two voxels while the Chebyshev polynomial method uses far more data points. Since the noise is assumed zero mean and independent it will effect less the more data points that are used. For no or low noise ratios the proposed polynomial model perform worse then the finite difference method. This is likely due to the extension from $2 \mathrm{D}$ to $3 \mathrm{D}$ computational domain. In a volume it is more likely that light from other particles influence the estimation. The false positive ratio is around $1 \%$ so it is very unlikely that noise is mistaken for a particle. This is due to the fact that noise rarely originate from a geometrical point and hence does not have the behavior of an outwards propagating wavefront. Therefore it does not have the same change in wavefront curvature as a particle.

The false negative ratio increased to around 5\% when using the Chebyshev model compared to $3 \%$ when using finite difference. A closer study of the undetected particles shows two main reasons for the increase. The first reason is inaccuracy in the course estimation. The model searches for a zero-crossing of $a_{200}$ close to the point obtained from the course estimation. So if that estimation is very inaccurate the model never finds the zero-crossing. The second reason is that particles that are located near the edges of the reconstructed volume do not use the same number of data points, since parts of the computational domain is shifted outside the reconstruction. This can cause the model to behave inaccurately.

The performance is also compared to the total sum of gradients method, which is commonly used to determine particles axial positions $[25,26]$. Applying the method to the simulated data set shows that the sum of gradients method results in a small offset from the true axial position, around $3 \mu \mathrm{m}$, which is still inside the particle. The standard deviation for the different noise levels showed a slight increase ranging from $7-12 \mu \mathrm{m}$ compared to $5-10 \mu m$ for the polynomial fit. The two methods hence show comparable noise susceptibility.

The results from the experimental measurements are presented in Fig.8. This figure shows the error histogram, after outliers have been removed. The error seems to have a Gaussian

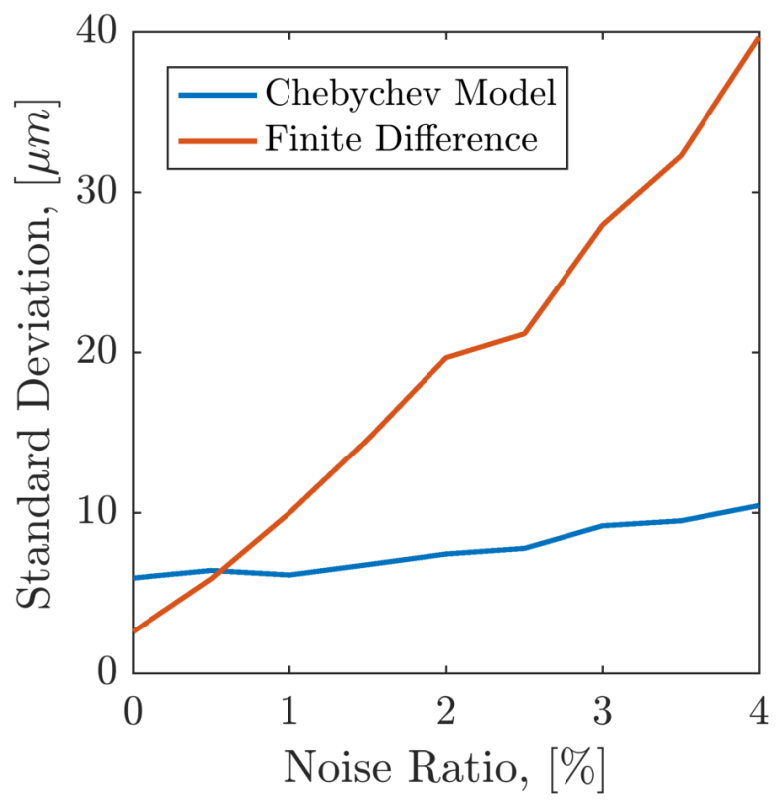

Fig. 7. Simulated standard deviations of axial position error using both Chebyshev model (blue) and finite difference method (red).

profile where the standard deviation is $\sigma=13 \mu \mathrm{m}$, which is an improvement compared with the finite difference method where the standard deviation was $18 \mu \mathrm{m}$ [21]. Even though the standard deviation is decreased it is still higher than for the simulations. From Fig.7, the expected standard deviation would be around $7 \mu \mathrm{m}$. This can depend on several factors. In the simulations we assume only single scattering and ignore any effects from the finite size of the silicon cube. For a single particle scattered light from other particles can be viewed as noise. It is possible that these effects influence the results. Also aspects like mechanical and thermal stability in the experimental setup influences the results. Still the standard deviation is in the same order of magnitude as the diameter of the particle. It is therefore likely that the measured position is inside or in the close vicinity of the particle.

Finally a brief discussion of the parametrization process is in order. Consider the errors presented in Fig. 4 . For $n=4$ the error is negligible. However, since the coefficient used as a metric is a second order term, higher terms are redundant due to the orthonormal properties of the base functions. This means adding higher order terms should not change lower order coefficients. The biggest error reduction is from $n=1$ to $n=2$. This is not a surprise since a spherical wave over a small aperture can be approximated by a second order term. This is also why a second order term $a_{200}$ is used as a metric. From Fig. 5 it is obvious that only $a_{200}$ can be used as a metric since $a_{020}$ is shifted along the optical axis. These coefficients represent quadratic terms parallel and perpendicular to the scattering plane respectively. The wavefront parallel to the scattering plane is modulated by the Lorenz-Mie scattering coefficients, while the perpendicular direction is not. The modulation distort the wavefront in the parallel direction causing $a_{020}$ to become zero at a shifted position, while $a_{200}$ is unaffected. This effect has previously been reported by $\mathrm{Pu}$ and Meng [27]. They related the modulation effects to aberrations for different observation directions. These 


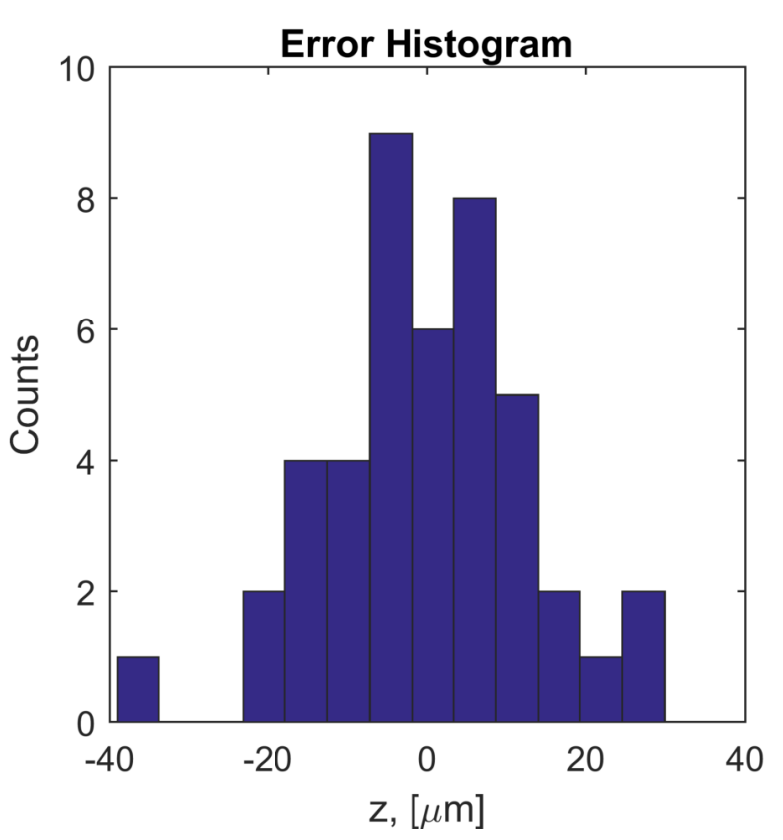

Fig. 8. Histogram of experimental error. The error is approximately zero mean with a standard deviation of $12.8 \mu \mathrm{m}$.

effects are important to consider when interpreting results from a certain observation direction. A future extension of the proposed polynomial method to include non-spherical particles could be possible. Non-spherical particles do in general have a dependence on the perpendicular direction as well [23], so the modulation affects needs to be handled. This is something that could be addressed in future work. Modulation effects can also be introduced by the reference wave. If the reference is a curved wave instead of a plane wave the curvature will modulate the phase response. It is therefore important to ensure the quality of the reference wave.

\section{CONCLUSIONS}

In this paper a parameterized model based on Chebyshev polynomials is introduced to estimate the wavefront curvature. It is shown that the phase anomaly around a scattering particle can be modeled by a three dimensional Chebyshev polynomial of degree $n=4$. To find this degree the mean error per voxel is evaluated. The polynomial coefficients holds information about the particle position and by using simulations the behavior of all obtained coefficients is studied. It is found that the coefficient $a_{200}$ becomes zero in the axial plane where the particle is located. This coefficient corresponds to the quadratic term perpendicular to the scattering plane. By evaluating this coefficient at different positions and searching for the axial plane where it becomes zero it is possible to find the true particle position. From simulations this method has standard deviations between $6-10 \mu \mathrm{m}$. This should be compared to the standard deviation using the finite difference method previously reported, that were $3-39 \mu \mathrm{m}$ for the same conditions. The Chebyshev polynomial model perform better in higher noise ratios since the noise in individual voxels do not affect the result to the same degree. For no or very low noise the worse performance is due to the extension of computational domain from $2 \mathrm{D}$ to $3 \mathrm{D}$. In a volume light from other particles is more easily introduced in the computation. Experimental results also shows improvement when using this method, from $18 \mu \mathrm{m}$ to $13 \mu \mathrm{m}$. This improvement is not as big as the improvement expected from simulations, however ideal conditions in simulations are rarely obtainable.

Funding. Swedish Research Council (VR) (621-2014-4906)

\section{REFERENCES}

1. D. Gabor, "A new microscopic principle," Nature 161, 777-778 (1948).

2. J. W. Goodman and R. W. Lawrence, "Digital image formation from electronically detected holograms," Applied Physics Letters 11, 77-79 (1967).

3. H. Meng, G. Pan, Y. Pu, and S. H. Woodward, "Holographic particle image velocimetry: from film to digital recording," Measurement Science and Technology 15, 673-685 (2004).

4. J. Sheng, E. Malkiel, and J. Katz, "Digital holographic microscope for measuring three-dimensional particle distributions and motions," Appl. Opt. 45, 3893-3901 (2006).

5. M. J. Berg and S. Holler, "Simultaneous holographic imaging and lightscattering pattern measurement of individual microparticles," Opt. Lett. 41, 3363-3366 (2016).

6. M. J. Berg, N. R. Subedi, and P. A. Anderson, "Measuring extinction with digital holography: nonspherical particles and experimental validation," Opt. Lett. 42, 1011-1014 (2017).

7. O. Kemppinen, Y. Heinson, and M. Berg, "Quasi-three-dimensional particle imaging with digital holography," Appl. Opt. 56, F53-F60 (2017).

8. Y. Wu, M. Brunel, R. Li, L. Lan, W. Ao, J. Chen, X. Wu, and G. Gréhan, "Simultaneous amplitude and phase contrast imaging of burning fuel particle and flame with digital inline holography: Model and verification," Journal of Quantitative Spectroscopy and Radiative Transfer 199, 2635 (2017).

9. Y. Wu, L. Yao, X. Wu, J. Chen, G. Gréhan, and K. Cen, "3d imaging of individual burning char and volatile plume in a pulverized coal flame with digital inline holography," Fuel 206, 429-436 (2017).

10. S. ichi Satake, Y. Yonemoto, T. Kikuchi, and T. Kunugi, "Detection of microbubble position by a digital hologram," Appl. Opt. 50, 5999-6005 (2011).

11. J. W. Goodman, Introduction to Fourier optics (McGraw-Hill, New York, 1996), 2nd ed.

12. N. Verrier and M. Atlan, "Off-axis digital hologram reconstruction: some practical considerations," Appl. Opt. 50, H136-H146 (2011).

13. M. Born and E. Wolf, Principles of optics : electromagnetic theory of propagation, interference and diffraction of light (Cambridge University Press, Cambridge, 1999), 7th ed.

14. Y. S. Bae, J. I. Song, and D. Y. Kim, "Volumetric reconstruction of Brownian motion of a micrometer-size bead in water," Optics Communications 309, 291-297 (2013).

15. J. K. Abrantes, M. Stanislas, S. Coudert, and L. F. a. Azevedo, "Digital microscopic holography for micrometer particles in air." Applied optics 52, A397-409 (2013).

16. L. Wilson and R. Zhang, "3D Localization of weak scatterers in digital holographic microscopy using Rayleigh-Sommerfeld back-propagation," Optics Express 20, 16735 (2012).

17. G. Pan and H. Meng, "Digital holography of particle fields: reconstruction by use of complex amplitude," Applied Optics 42, 827-833 (2003).

18. J. de Jong and H. Meng, "Digital holographic particle validation via complex wave," Applied optics 46, 7652-7661 (2007).

19. F. Soulez, L. Denis, C. Fournier, Éric Thiébaut, and C. Goepfert, "Inverse-problem approach for particle digital holography: accurate location based on local optimization," J. Opt. Soc. Am. A 24, 11641171 (2007).

20. J. Fung, K. E. Martin, R. W. Perry, D. M. Kaz, R. McGorty, and V. N. Manoharan, "Measuring translational, rotational, and vibrational dynamics in colloids with digital holographic microscopy," Opt. Express 19, 8051-8065 (2011).

21. J. Öhman and M. Sjödahl, "Off-axis digital holographic particle positioning based on polarization-sensitive wavefront curvature estimation," Appl. Opt. 55, 7503-7510 (2016). 
22. F. C. Cheong, B. J. Krishnatreya, and D. G. Grier, "Strategies for three-dimensional particle tracking with holographic video microscopy." Optics express 18, 13563-13573 (2010).

23. C. F. Bohren and D. R. Huffman, Absorption and scattering of light by small particles (John Wiley \& Sons, 1998).

24. M. Abramowitz and I. A. Stegun, Handbook of mathematical functions with formulas, graphs, and mathematical tables, vol. 9 (Dover, New York, 1972).

25. Y.-S. Choi, K.-W. Seo, M.-H. Sohn, and S.-J. Lee, "Advances in digital holographic micro-ptv for analyzing microscale flows," Optics and Lasers in Engineering 50, 39 - 45 (2012). Advances in Flow Visualization.

26. P. Memmolo, L. Miccio, M. Paturzo, G. D. Caprio, G. Coppola, P. A. Netti, and P. Ferraro, "Recent advances in holographic 3d particle tracking," Adv. Opt. Photon. 7, 713-755 (2015).

27. $\mathrm{Y}$. Pu and $\mathrm{H}$. Meng, "Intrinsic aberrations due to mie scattering in particle holography," J. Opt. Soc. Am. A 20, 1920-1932 (2003). 